The Astrophysical Journal, 321:L17-L21, 1987 October 1

( ) 1987. The American Astronomical Society. All rights reserved. Printed in U.S.A.

\title{
DISCOVERY OF A PROBABLE BINARY QUASAR ${ }^{1}$
}

\author{
S. DJORGOVSKI ${ }^{2}$ \\ Harvard-Smithsonian Center for Astrophysics \\ R. PERLEY \\ National Radio Astronomy Observatory \\ G. MEYLAN \\ European Southern Observatory \\ AND \\ P. MCCARThY \\ Astronomy Department, University of California at Berkeley \\ Received 1987 May 8; accepted 1987 July 13
}

\begin{abstract}
We report discovery of a pair of quasars at a redshift of 1.345 , separated by 4.2 in projection, apparently associated with the radio source PKS $1145-071$. The optical intensity ratio of the two images is approximately 2.5. The spectra are very similar, and all emission lines except for the C IV $\lambda 1549$ have the same equivalent widths, within the error bars. The redshift difference based on the $\mathrm{C}$ IV line alone, which is the strongest feature, and on the cross-correlation of the two spectra including the C IV line, is approximately $250 \pm 100 \mathrm{~km} \mathrm{~s}^{-1}$ in the rest frame. If this discrepant emission line is excluded, the redshift difference drops to approximately $100 \pm 100 \mathrm{~km} \mathrm{~s}^{-1}$, and is consistent with zero. Thus, on the basis of the optical data alone, we would have concluded that this double QSO is another example of gravitational lensing. However, the radio source is single and unresolved at all VLA frequencies, and positionally coincident with the brighter of the two optical images. The fainter of the two optical components is at least a factor of 500 fainter in the radio. This dramatic difference in $L_{\mathrm{OPT}} / L_{\mathrm{RADIO}}$ between the two components suggests that the system is a genuine binary quasar, and the first such well-documented case. Assuming that the two QSO's are gravitationally bound, we derive a lower limit to the total mass of the system of $\sim 10^{11} h^{-1} M_{\odot}$; this is the first such direct determination of a quasar mass. The close projected separation of the pair $\left(\sim 20 h^{-1} \mathrm{kpc}\right)$ and the small velocity difference are suggestive of an interaction, which may be causally related to the QSO activity, and of the existence of a parent group or cluster, which would be a very interesting find in itself. In the view of this discovery, it is plausible that some other, previously reported gravitational lenses may be really pairs of physically distinct quasars.
\end{abstract}

Subject headings: gravitation - quasars — radio sources: general

\section{INTRODUCTION}

The subject of gravitational lensing is one of the most vigorous and fastest growing in modern extragalactic astronomy. Good recent reviews of the field are given by Turner (1984) and Burke (1986). Theoretical discussions are fluorishing, but the data are scarce: there are only a few systems for which the gravitational lensing interpretation is now reasonably well established, viz., 0957+561 (Walsh, Carswell, and Weymann 1979), 1115 + 080 (Weymann et al. 1980), 2016 +112 (Lawrence et al. 1984,) and probably 2237+030 (Huchra et al. 1985). In two other possible cases, $2345+007$ (Weedman et al. 1982) and $1635+267$ (Djorgovski and Spinrad 1984), to this date there is no detection of lensing objects, and thus they should be regarded as tentative true

\footnotetext{
${ }^{1}$ Based in part on observations made at the Multiple Mirror Telescope Observatory, a joint facility of the Smithsonian Institution and University of Arizona, and on observations made at the European Southern Observatory, La Silla, Chile.

${ }^{2}$ Society of Fellows, Harvard University.
}

pairs of physically distinct quasars. Many new candidates are expected from the VLA gravitational lens survey (cf. Hewitt et al. 1987). A number of systems were proposed in the literature as examples of gravitational (macro- or micro- or mega-) lensing, sometimes without a really adequate observational evidence.

As a constructive reaction to this trend, several authors proposed that some of the claimed "gravitational lens" systems are actually physical pairs of quasars with small separations (Phinney and Blandford 1986; Bahcall, Bahcall, and Schneider 1986). This is a natural expectation based on the observed degree of galaxy clustering at low redshifts, and reasonable extrapolations to large redshifts. There are, in fact, several pairs of quasars known, with projected angular and redshift separations indicative of membership in large clusters or superclusters (e.g., 1146+111; Hazard, Arp, and Morton 1979; 0952 + 698, Burbidge et al. 1980; 1037-271, Sargent and Steidel 1987; see also Shaver 1985, and references therein). However, there are no definite close physical quasar pairs 
currently known at any redshift. In the Letter we report a discovery of a high-redshift QSO pair which may be the first binary quasar known.

The radio source OM-076 was first identified with a blue, 17.5 mag stellar object on the Palomar Sky Survey by Radivich and Kraus (1971), and then "rediscovered" as PKS $1145-071$ by Bolton, Shimmins, and Wall (1975), who reaffirmed the optical identification. The spectroscopy of the object by Wilkes et al. (1983) and Wilkes (1986) identified it as a QSO with $z=1.345$. VLBI measurements of the radio source were published by Preston et al. (1985). There was no mention by any author of the source's binary structure. The QSO was selected for an optical imaging search for gravitational lenses (Djorgovski et al., in preparation) because a marginal elongation was visible on the finding chart published by Bolton et al.

\section{THE OBSERVATIONAL RESULTS}

We obtained initial images of the field on the night of UT 1986 December 29, using a RCA $320 \times 512$ (no. 5) CCD mounted at the Cassegrain focus $(f / 8.01)$ of the ESO $2.2 \mathrm{~m}$ telescope. The effective pixel size was $0^{\prime \prime} 363$. The conditions were marginally nonphotometric, and the seeing $\mathrm{FWHM} \approx$ 1 1.5. One $B$ and one $V$ exposure of $300 \mathrm{~s}$ each were obtained, and processed using standard techniques. The $V$ frame is shown in Figure 1 (Plate L2). We obtain for the separation of the two components:

$$
\Delta \alpha_{\mathrm{A}-\mathrm{B}}=-3^{\prime \prime} .3 \pm 0^{\prime \prime} .1, \quad \Delta \delta_{\mathrm{A}-\mathrm{B}}=+2^{\prime \prime} .6 \pm 0^{\prime \prime} .1,
$$

which corresponds to the separation of 4 ".2 in the direction $\mathrm{PA}=128^{\circ}$. Our magnitude zero points are uncertain (approximate magnitude of the QSO $A$ is $\sim 18$ ), but we can derive accurate intensity ratios for the two images, $I_{\mathrm{A}} / I_{\mathrm{B}}=$ $2.15 \pm 0.15$ in the $B$ band, and $2.7 \pm 0.1$ in the $V$ band.

Spectra of the two components were obtained on the nights of UT 1987 January 3 and 4, by using the Reticon spectrograph (Latham 1982) on the Multiple Mirror Telescope at Mount Hopkins. We used the low-resolution (300 lines $\mathrm{mm}^{-1}$ ) grating, and $2^{\prime \prime} \times 3^{\prime \prime}$ entrance apertures. The seeing was fairly good, but transparency variable, which prevented an adequate flux calibration of the data. The spectra confirmed immediately that both objects $\mathrm{A}$ and $\mathrm{B}$ are quasars, at apparently the same redshift. We observed both objects in the left and right channels of the spectrograph, in order to improve the sky subtraction, and interleaved observations of the two objects in order to minimize the systematic errors due to any possible flexure of the spectrograph. Exposure of the $\mathrm{He}-\mathrm{Ne}-\mathrm{Ar}-\mathrm{Cd}$ comparison source were obtained before, after, and on January 4 also between the pairs od QSO exposures. The resultant wavelength calibration has a r.m.s. of $\sim 1 \AA$, and systematic errors smaller than that, which we checked from the measurements of night sky lines. The total integrations for the QSO's A and B were $600 \mathrm{~s}$ and $2000 \mathrm{~s}$ respectively on January 3, and $960 \mathrm{~s}$ and $2160 \mathrm{~s}$ on January 4. The spectra are shown in Figure 2.

The spectra appear very similar, except for the C IV $\lambda 1549$ line, which clearly has a larger equivalent width in the QSO
A. The redshift of this quasar, based on the $\mathrm{C}$ IV line alone is $z_{\mathrm{A}}=1.345 \pm 0.001$. The observed equivalent widths of other emission lines are same for the two QSOs to within the measurement errors; our values are in good agreement with the measurements by Wilkes (1986).

Considerable care was given to the measurement of velocity difference between the two QSOs: we did wavelength calibration in two different ways, measured redshift difference from the strong C IV line, and from the cross-correlation of spectra, for the two nights separately, and using different portions of the spectra. We employed two different centering methods for the emission lines and cross-correlation peaks (Gaussian least-squares fit to the peak center, and convolution with a Gaussian derivative kernel). Different methods and variation of parameters enabled us to estimate fairly our internal errors. The cross-correlation method for QSO pairs was described by Djorgovski and Spinrad (1984), and Huchra (1986), and references therein. An example of the cross-correlation of the two spectra is shown in Figure 3. From the centering of the C IV line only, we obtain $\Delta z_{\mathrm{A}-\mathrm{B}}=0.001 \pm 0.003$, independently for both nights, corresponding to the rest frame velocity difference $\Delta v_{A-B}^{\text {rf }}=300 \pm 800 \mathrm{~km} \mathrm{~s}^{-1}$. A more accurate method, cross-correlation using complete spectra (still dominated by the discrepant $C$ IV line, which is the strongest feature), in wavelength range 3200-7000 $\AA$, gives $\Delta z_{\mathrm{A}-\mathrm{B}}=$ $(9.3 \pm 2.7) \times 10^{-4}$, or $\Delta v_{\mathrm{A}-\mathrm{B}}^{\mathrm{rf}}=280 \pm 80 \mathrm{~km} \mathrm{~s}^{-1}$ for the January 3 data, and $\Delta z_{\mathrm{A}-\mathrm{B}}=(6.5 \pm 3.8) \times 10^{-4}$, or $\Delta v_{\mathrm{A}-\mathrm{B}}^{\mathrm{rf}}$ $=200 \pm 110 \mathrm{~km} \mathrm{~s}^{-1}$ for the January 4 data, which have somewhat more reliable wavelength calibration and more signal. Similar numbers are obtained if one uses the wavelength range 3200-4800 $\AA$. However, if we exclude the portion dominated by the discrepant $\mathrm{C}$ IV line, and use the data in the range $3800-7000 \AA$, the difference drops to $\Delta z_{\mathrm{A}-\mathrm{B}}=$ $(4.1 \pm 3.1) \times 10^{-4}$, or $\Delta v_{\mathrm{A}-\mathrm{B}}^{\text {rf }}=120 \pm 90 \mathrm{~km} \mathrm{~s}^{-1}$ for the January 4 data (the January 3 data do not have enough signal to do this test).

Short observations of PKS 1145 - 071 were obtained with the Very Large Array (VLA) ${ }^{3}$ on the night of UT 1987 January 9. Data were taken at $90,20,6.2$, and $1.3 \mathrm{~cm}$, but in view of the configuration available (C), only the data taken at 2 and $1.3 \mathrm{~cm}$ had sufficient resolution to clearly determine whether the object contained two sources of emission, or one. Thus, the majority of the available time was spent at $2 \mathrm{~cm}$. All the data were calibrated by short observations of nearby point sources. Subsequently, the standard self-calibration routines were applied, and the resulting maps have excellent dynamic range, $\sim 2700: 1$ at $2 \mathrm{~cm}$ and $6 \mathrm{~cm}$, and $\sim 200: 1$ at $1.3 \mathrm{~cm}$. The $2 \mathrm{~cm}$ map is shown in Figure 4. The source is unresolved at all frequencies, and there is no trace of a secondary image. The radio spectrum shows a sharp inversion at the high frequencies.

The best-fit radio position of the source (epoch 1950.0) is:

$$
\alpha=11^{\mathrm{h}} 45^{\mathrm{m}} 18^{\mathrm{s}} .29, \quad \delta=-07^{\circ} 08^{\prime} 00.56^{\prime \prime}
$$

\footnotetext{
${ }^{3}$ The VLA/NRAO is operated by the Associated Universities, Inc., under a contract with the NSF.
} 


\section{QQ 1145-071}

is


FIG. 1. $-V$ band CCD image of the PKS $1145-071$ field, obtained at ESO. The field size is 115" by 184", with north to the top, and east to the left. The two QSOs are labeled as A and B.

DJORGOVSKI et al. (see 321, L18). 


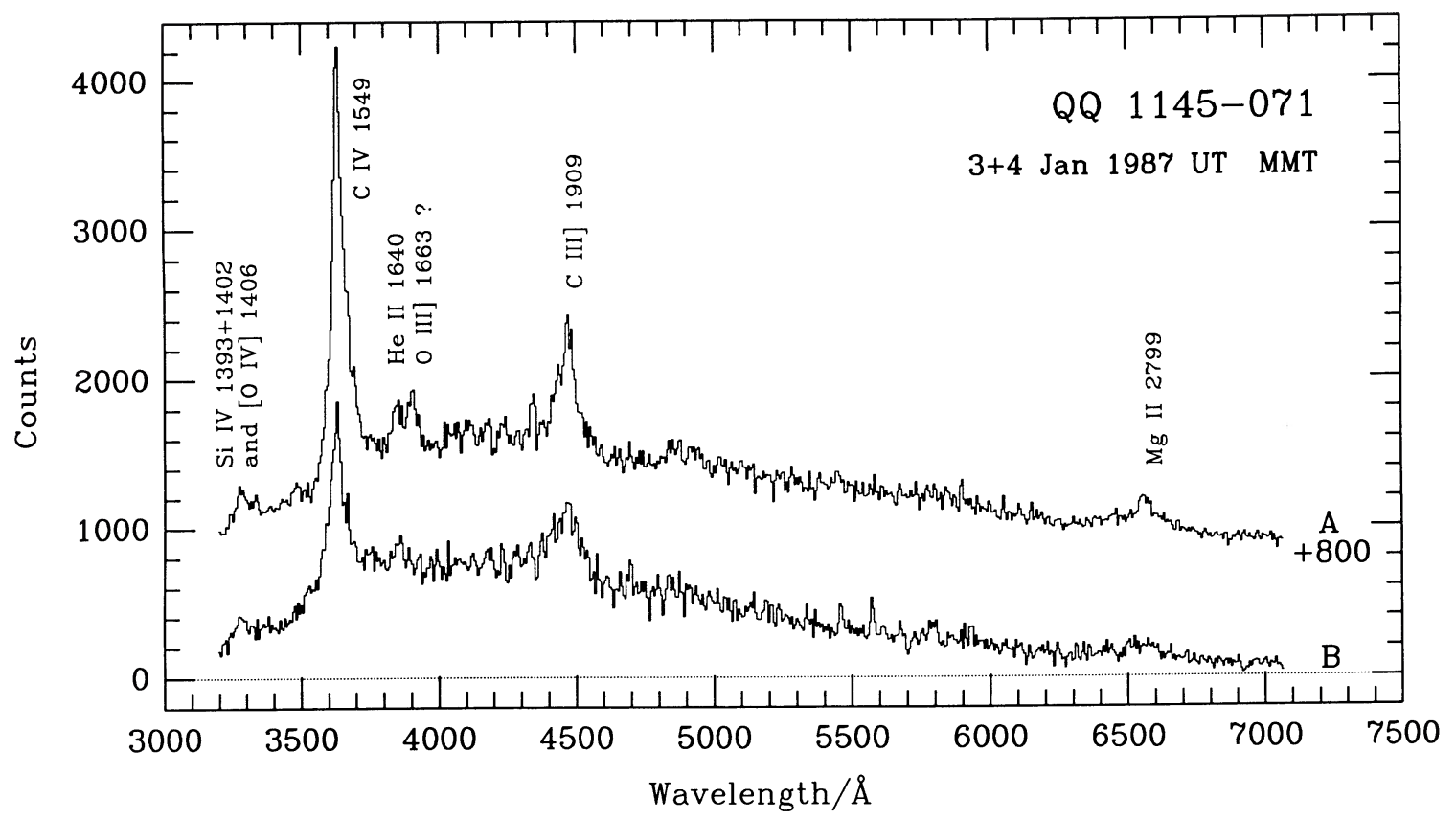

FIG. 2. - The spectra of the two quasars, obtained at the MMT: QSO A on the top, QSO B on the bottom. The spectrum of the QSO A was shifted up by 800 units for clarity. Both spectra were rebinned to $6 \AA$ bins (the instrumental resolution is FWHM $\sim 12 \AA$ ). The relative flux scale is arbitrary.

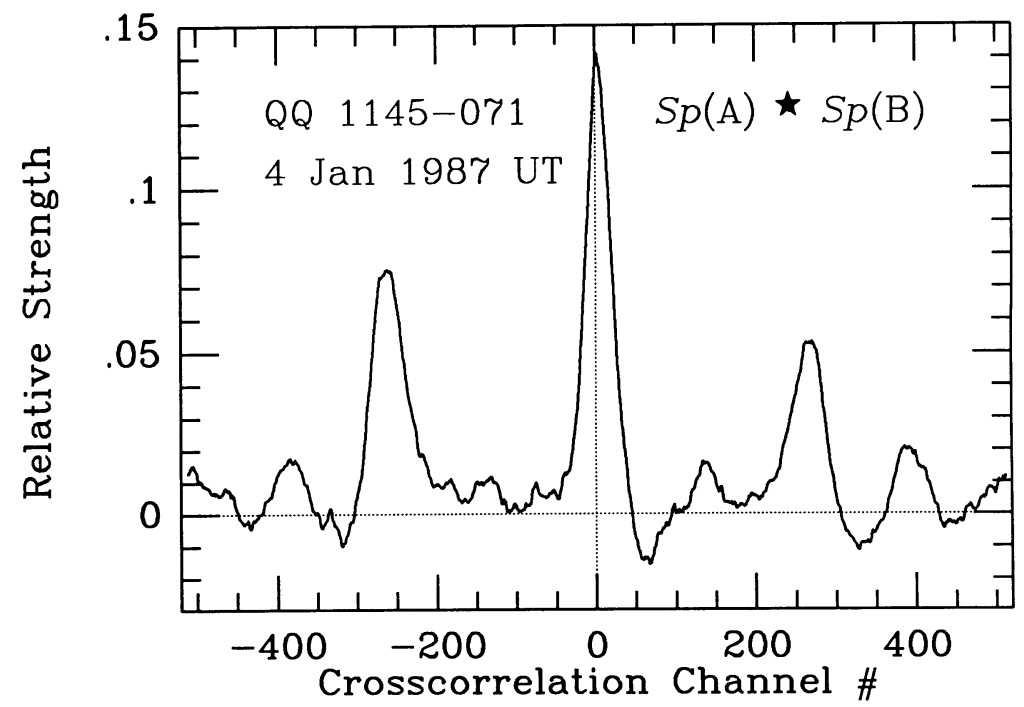

FIG. 3. - Cross-correlation of the spectra of the two QSOs, using the January 4 data in the wavelength region 3200-7000 A. One channel corresponds to $\Delta z=3.3 \times 10^{-4}$.

with errors of 0 ".05 in each coordinate. We obtained the optical position of the brighter of the two QSOs from the independent measurements of both red (E) and blue (O) prints of the Palomar Sky Survey, by using the Center for Astrophysics dual axis measuring machine. Some 20 SAO stars were used to establish the coordinate system. The final mean position (epoch 1950.0) is:

$$
\alpha=11^{\mathrm{h}} 45^{\mathrm{m}} 18^{\mathrm{s}} .30, \quad \delta=-07^{\circ} 08^{\prime} 01.05^{\prime \prime},
$$

with errors of 0 ".6 in each coordinate. This measurement pertains to the brighter QSO, A, but the presence of the fainter component B is likely to "pull" the measured center slightly to south and east, exactly in the sense in which the radio and the optical positions differ. As an additional test, we determined equatorial positions of four nearby stars, visible on our CCD frames, and used them as "secondary" standards to determine the coordinates of the two QSOs. We again found that the QSO $A$ is coincident with the radio 


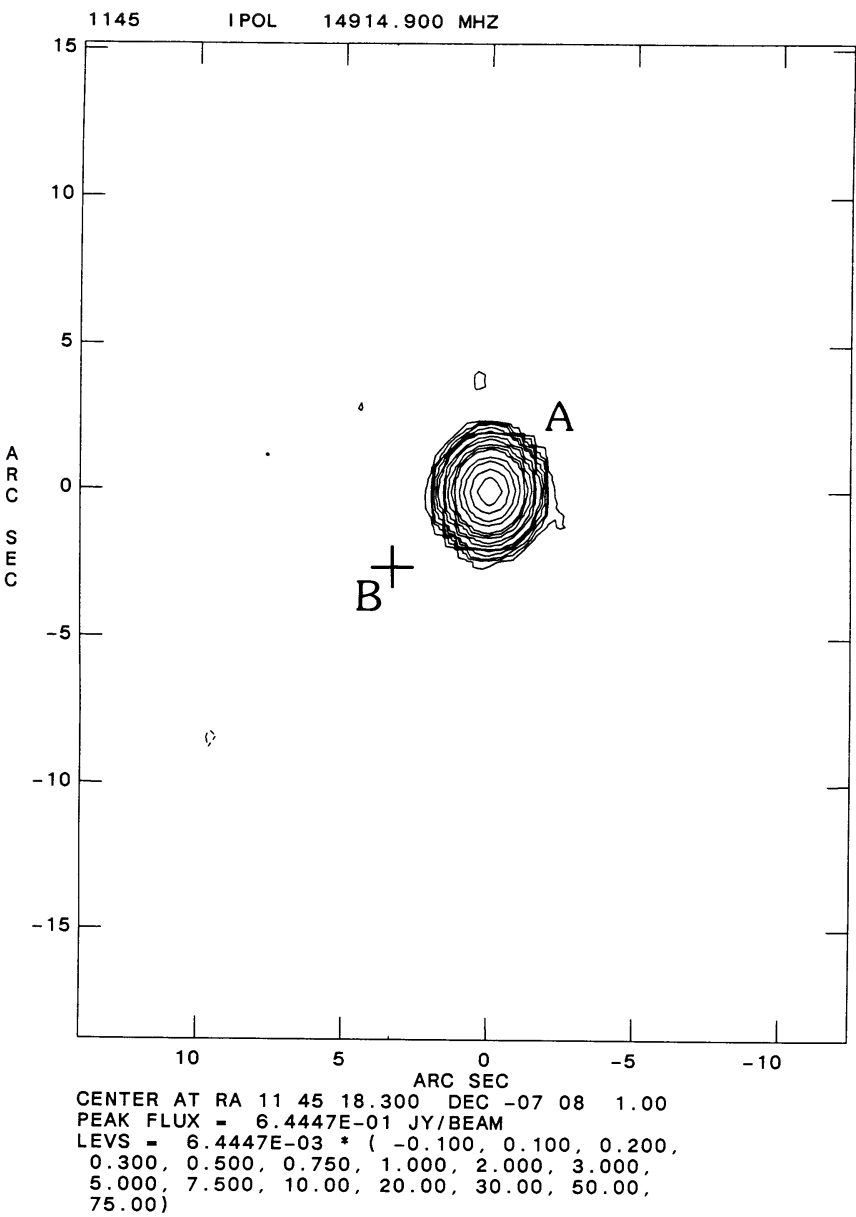

FIG. 4.-Radio map of PKS 1145-071, obtained at VLA at $2 \mathrm{~cm}$, in the $\mathrm{C}$ configuration. The position of the QSO B is marked with the cross. The residual noise in this map is $0.23 \mathrm{mJy}$.

source to within $0^{\prime \prime} 7 \pm 0$.'7. Our measurements are also in an excellent agreement with both radio and optical positions published by Bolton, Shimmins, and Wall (1975), and Véron et al. (1976), as well as with the coarser positions published by Radivich and Kraus (1971) and Preston et al. (1985). There is thus little doubt that the radio source is positionally associated with the QSO A.

\section{DISCUSSION AND CONCLUSIONS}

To interpret the data in terms of physical scales, we note that in a Friedmann cosmology with $H_{0}=100 \mathrm{~km} \mathrm{~s}^{-1} \mathrm{Mpc}^{-1}$, $\Lambda_{0}=0$, and $q_{0}=0$, and assuming the redshift of galaxy formation $z_{\mathrm{gf}}=5$, the distance modulus to the system is $(m-M)=44.14$, the age $2.54 \mathrm{Gyr}$, and the projected separation of 4.2 corresponds to $25.0 \mathrm{kpc}$. If we substitute $q_{0}=1 / 2$, these numbers become $43.44,1.37 \mathrm{Gyr}$, and 18.1 kpc, respectively.

The imaging and spectroscopic data are consistent with the interpretation of the pair as a gravitational lens; the evidence being as strong as for any of the previously claimed lenses at a comparable stage of investigation. The difference in the equivalent widths of the C IV line, its shape, and thus the redshift difference, could be easily attributed to the intrinsic variation (which can occur on a time scale of a few months) plus the time delay between the two images (which may be of the order of 1 or $2 \mathrm{yr}$ ). The apparent optical properties of the system did not appear very different from the "classic" gravitational lens $0957+561$. However, this evidence is neither compelling nor conclusive, and is also consistent with the interpretation of the pair as a physical binary quasar.

The crucial evidence comes from our radio maps: the intensity ratio on $\mathrm{cm}$ wavelengths is at least several hundred, which should be compared to the optical intensity ratio of $\sim 2.5$. In order to salvage the gravitational lens hypothesis, we would require a dramatic $L_{\mathrm{OPT}} / L_{\mathrm{RADIO}}$ variability on the time scales corresponding to the path delay $(\sim 1 \mathrm{yr})$, at a level never before observed for an extragalactic source. Gravitational microlensing may help: the region from which the optical continuum originates should be an order of magnitude smaller than the region from which the radio continuum originates, and could be magnified more in the QSO B image. However, it is unlikely that this hypothetical phenomenon would produce a sufficient differential magnification for the radio continuum and the optical continuum, since numerical studies (e.g., by Schneider and Weiss 1987) indicate that the microlensing magnification ratio may amount to a factor of 10 , but certainly not a few hundred. Besides, the physical sizes of the broad-line region and the radio continuum region are expected to be roughly comparable, and we do not see drastic difference in the strength of emission lines. Preliminary optical monitoring indicates no strong variability on the scales of a few months ( $R$. Schild, private communication), and there is no indication of a major change between the Sky Survey plate (taken some $30 \mathrm{yr}$ ago), and our CCD images. We thus conclude the QSO pair $1145-071$ is most likely a genuine binary quasar.

It is tempting to compare this system with two other QSO pairs, $2345+007$, and $1635+267$, both of which are radioquiet, and for both of which the spectroscopic and imaging evidence for gravitational lensing are about equally good as in the case of 1145-071 here (cf. Shaver, Wampler, and Cristiani 1987). For both of those systems there is no obvious lensing cluster and/or galaxy to very faint magnitude levels (Tyson et al. 1986; Djorgovski et al., in preparation). Pending further studies, we should leave these two cases open.

A physical binary quasar should be an interesting object to study, as it may provide us with some clues about the origin and maintenance of QSO activity. Evidence for tidal interactions is often indicated in the low-redshift QSO-galaxy associations, and even proposed as a possible trigger of the QSO activity (cf. the reviews by Balick and Heckman 1982, Hutchings 1983, or Stockton 1986, and references therein). Tidal shocks may facilitate a runaway gravitational collapse of the central cluster in participating galaxies, and thus actually form the central "engine," or feed more stars into it if it already exists. Galaxy collisions also provide plausible means of transporting the ISM fuel to the central engines. The projected separation and the velocity difference between the two components of $1145-071$ are consistent with a tidal encounter.

Assuming that the two QSOs are gravitationally bound, and that there are no other very massive objects in their immediate proximity, we can use their projected separation 
and the velocity difference to place a lower limit to the total mass of the system. From the equality of the kinetic and potential energies, and independently of the shape of the orbit, the following equation should apply:

$$
M_{\mathrm{A}}+M_{\mathrm{B}}=\frac{R v^{2}}{2 G},
$$

where $M_{\mathrm{A}}$ and $M_{\mathrm{B}}$ are the masses of the quasars; $R$, their three-dimensional separation; and $v$, their three-dimensional mutual velocity. Now, the projected separation and the observed radial velocity difference are lower limits to $R$ and $v$, independent of the inclination and phase of the orbit. If we adopt $R_{\min } \approx 20 \mathrm{kpc}$, and $v_{\min } \simeq 200 \mathrm{~km} \mathrm{~s}^{-1}$, we derive $M_{\mathrm{A}}+M_{\mathrm{B}} \simeq 10^{11} M_{\odot}$ as a lower limit, which is a reasonable value for normal galaxies. Allowing for the projection effects would suggest a true value several times larger than that. This is, to our knowledge, the first such direct determination of a quasar mass.

Future high-resolution absorption-line spectroscopy of this system may be used to probe the physical state and dynamics of the ionized gas around the QSOs. Studies of correlated absorption lines in the spectra of the two QSOs, blueward of the carbon or magnesium lines from the ground, or Ly $\alpha$ with the Hubble Space Telescope, can place new and better limits on the physical size of intergalactic clouds (cf. Foltz et al. 1984).

Another exciting possibility is that this pair is pointing toward a high-redshift galaxy cluster: if quasars are rare events, then two quasars are suggestive of a high galaxy density. Mere existence of right clusters at such large redshifts provides an interesting timing constraint for the theories of large-scale structure formation. Studies of "normal" galaxies in this hypothetical cluster should be extremely valuable for the investigations of galaxy evolution at large look-back times.

We would like to thank the staffs of MMT, ESO, and VLA observatories for their able help during our observing runs, and to John Huchra for the guidance at the MMT. We also thank Susan Tokarz for her help in MMT data reductions. Rudy Schild obtained some early CCD frames of the field. David Morabito kindly provided some unpublished information of the VLBI measurements. We acknowledge constructive comments of an anonymous referee. S. D. acknowledges a partial support from Harvard University.

\section{REFERENCES}

Bahcall, J., Bahcall, N and Schneider, D. 1986, Nature, 323, 515.

Balick, B., and Heckman, T. 1982, Ann. Rev. Astr. Ap., 20, 431.

Bolton, J., Shimmins, A., and Wall, J. 1975, Australian J. Phys. Suppl., 34, 1.

Burbidge, E. M., Junkkarinen, V., Koski, A., Smith, H. E., and Hoag, A. 1980, Ap. J. (Letters), 242, L55.

Burke, B. 1986, in IAU Symposium 119, Quasars, ed. G. Swarup and V. Kapahi (Dordrecht: Reidel), p. 517.

Djorgovski, S., and Spinrad, H. 1984, Ap. J. (Letters), 282, L1.

Foltz, C., Weymann, R., Röser, H.-J., and Chaffee, F. 1984, Ap. J. (Letters), 281, L1.

Hazard, C., Arp, H., and Morton, D. 1979, Nature, 282, 271.

Hewitt, J., Turner, E., Burke, B., Lawrence, C., Bennett, C., Langston, G., and Gunn, J. 1987, in IAU Symposium 124, Observational Cosmology, ed. G. Burbidge, (Dordrecht: Reidel), in press.

Huchra, J. Gorenstein, M., Kent, S., Shapiro, I., Smith, G., Horine, E., and Perley, R. 1985, A.J., 90, 691.

Huchra, J. 1986, Nature, 323, 784

Hutchings J. 1983, Pub. A.S.P., 95, 799.

Latham, D. 1982, in IAU Colloquium 67, Instrumentation for Astronomy with Large Optical Telescopes, ed. C. Humphries (Dordrecht: Reidel), p. 259.

S. DJorgovskI: Center for Astrophysics, 60 Garden Street, Cambridge, MA 02138

P. MCCARThy: Astronomy Department, University of California, Berkeley, CA 94720

G. MEYLAN: European Southern Observatory, Karl-Schwarzschild-Strasse 2, D-8046 Garching bei München, Federal Republic of Germany

R. Perley: VLA/NRAO, P.O. Box 0, Socorro, NM 87801
Lawrence, C., et al. 1984, Science, 223, 46.

Phinney, S., and Blandford, R. 1986, Nature, 321, 569.

Preston, R., Morabito, D., Williams, J., Faulkner, J., Jauncey, D., and Nicolson, G. 1985, A.J., 90, 1599.

Radivich, M., and Kraus, J. 1972, A.J., 76, 683.

Sargent, W., and Steidel, C. 1987, Ap. J., in press.

Schneider, P., and Weiss, A. 1987, Astr. Ap., 171, 49.

Shaver, P. 1985, Astr. Ap., 143, 451.

Shaver, P., Wampler, J., and Cristiani, S. 1987, Nature, 327, 40.

Stockton, A. 1986, Ap. Space Sci., 118, 487.

Turner, E. 1984, Science, 223, 1255.

Tyson, J. A., Seitzer, P., Weymann, R., and Foltz, C. 1986, A.J., 91, 1274.

Véron, M., Véron, P., Adgie, R., and Gent, H. 1976, Astr. Ap., 47, 401.

Walsh, D., Carswell, R., and Weymann, R. 1979, Nature, 279, 381.

Weedman, D., Weymann, R., Green, R., and Heckman, T. 1982, Ap. J.

(Letters), 255, L5.

Weymann, R., et al. 1980, Nature, 285, 641.

Wilkes, B. 1986, M.N.R.A.S., 218, 331.

Wilkes, B., Wright, A., Jauncey, D., and Peterson, B. 1983, Proc. Astr. Soc. Australia, $5,2$. 